\title{
Research on butene oligomerization reaction over the hemicellulose modified HZSM-5
}

\author{
LI Chao ${ }^{1,2}$, WANG Hui ${ }^{1, \star}$, ZHU Shan-shan ${ }^{1,2}$, LIU Guang-bo ${ }^{1}$, WU Jin-hu ${ }^{1}$ \\ ${ }^{1}$ Key Laboratory of Biofuels, Qingdao Institute of Bioenergy and Bioprocess Technology, Chinese Academy of Sciences, Qingdao 266101, \\ China; \\ ${ }^{2}$ University of Chinese Academy of Sciences, Beijing 100049, China
}

\begin{abstract}
The HZSM-5 was synthesized with modifying by different contents of hemicellulose. The morphology, texture and acidity were characterized with XRD, SEM, $\mathrm{N}_{2}$ adsorption-desorption, $\mathrm{NH}_{3}$-TPD and Py-IR. Butene oligomerization over the modified HZSM-5 was tested. The results indicated that the HZSM-5 with $1.6 \%$ hemicellulose exhibited the best activity and stability on the butene oligomerization reaction. The conversion can maintain about $80 \%$ after $140 \mathrm{~h}$ on stream with the highest selectivities of the trimer, tetramer and diesel component in the products, which may be related with its high BET surface and mesoporous volume, and the strongest acidity.
\end{abstract}

Key words: hemicellulose; modified; HZSM-5; butene oligomerization

With the growth demand of oil and the less oil storage in China, the oil production is difficult to self-sufficiency and the shortage of oil has become an inevitable fact ${ }^{[1]}$. There is rich of coal with less oil and gas energy structure in China. Hence, reinforcing the clean and high utilization of coal, developing non-oil route to produce clean fuel has important strategic significance for producing environmental friendly liquid fuels as well as adjusting energy structure. On the other hand, with the progress of catalytic cracking and Fischer-Tropsch synthesis technology ${ }^{[2,3]}$, olefin resources are increasingly abundant. Furthermore, with the MTO process achieving milestone progress in China, developing coal chemical industry combined with the MTO/MTP process, and using the light olefin to produce high quality liquid fuels can be an important comprehensive route in the utilization of olefin downstream products ${ }^{[4,5]}$.

Oligomerization, refers to the process of a certain number of monomer olefin polymerized to the compound with one or more tectonic units connected to repeat ${ }^{[5]}$. The oligomerization of light olefins (ethene, propene and butene) is an important way to produce environmental friendly liquid fuels without sulfur and aromatics. As the difference of catalysts and reaction conditions, the polymerization degree, levels of branched chain and the proportion of gasoline and diesel components in products will also be different ${ }^{[6,7]}$. As early as
1935, oligomerization was already applied to chemical industry. Initially, the American UOP company used diatomite phosphate catalytic $\mathrm{C}_{4}$ or $\mathrm{C}_{3-4}$ fraction to have non-selective oligomerization with the main products of gasoline ${ }^{[8,9]}$. Until the 1980s, the MOGD technology developed by Mobile, using HZSM-5 zeolite as catalyst and $\mathrm{C}_{4}$ as raw material, made the oligomerization technology of producing diesel and/or gasoline products another great progress ${ }^{[10,11]}$. However, the oligomerization technology in China was dated from 1950s-60s and there was a big gap in operating equipment and preparing catalysts to reach international level ${ }^{[12]}$. With the rapid development of science and technology in recent years, the oligomerization process in China has also made new breakthroughs ${ }^{[5,13-15]}$. Though the olefins oligomerization technology has already been industrialized, developing and optimizing of catalysts are still the key to technological progress. The light olefin oligomerization is usually conducted on solid acid catalysts, such as solid phosphoric acid catalysts $^{[16]}$, zeolite catalysts ${ }^{[17]}$ and $\mathrm{Ni}$ supported zeolite catalysts $^{[18]}$. The solid phosphate catalyst is not very appreciated recently because of its easy clay, high degree branched chain of products, corrosion to equipment and not easy to regeneration ${ }^{[16]}$. At the same time, the zeolite catalysts are widely studied for their advantages of environmental friendly, easy to regeneration and the ability to

Received: 28-March-2017; Revised: 05-June-2017.

Foundation items: Supported by the Chinese Academy of Sciences Research Program (XDA07070302), the Applied Basic Research Programs of Qingdao(15-9-1-72-jch) and the Foundation of state Key Laboratory of high-efficiency Utilization of Coal and Green Chemical Engineering (2016-11).

*Corresponding author. Tel: 0532-80662764, E-mail: huiwangsun@ gmail.com.

Copyright (c) 2017, Institute of Coal Chemistry, Chinese Academy of Sciences. Published by Elsevier Limited. All rights reserved. 


\section{Chao et al / Journal of Fuel Chemistry and Technology, 2017, 45(9): 1088-1094}

regulate oil-gas ratio ${ }^{[19,20]}$, especially for the HZSM-5 zeolite with MFI three-dimensional channel structure and good acidity. As a result of the mesoporous and shape selective properties, it exhibits high coking resistance with products not easy to be branched. The ZSM-5 zeolite is regarded as the most promising catalyst for conversion of $\mathrm{C}_{3}$ and $\mathrm{C}_{4}$ alkenes to high quality liquid fuels ${ }^{[21,22]}$. In order to improve the catalytic ability of HZSM-5 to oligomerization, researches on modifying its structure with hierarchical channel and enhancing acidity have been widely reported ${ }^{[23,24]}$. Hemicellulose as the second most abundant renewable biomass is widespread in nature. The large number of hydroxyl and carboxylic groups on it provide a powerful basis structure for it as additive to modify the channel structure and acidity of HZSM-5 ${ }^{[25]}$.

In this work, based on the traditional hydro-thermal synthesis, hemicellulose was added to modify the properties of HZSM-5, to explore the influence of different contents of hemicellulose on HZSM-5. Butene oligomerization was as probe reaction to investigate the catalytic ability of modified HZSM-5 by different contents of hemicellulose. XRD, SEM, $\mathrm{N}_{2}$ adsorption/desorption, $\mathrm{NH}_{3}$-TPD and Py-IR are used to characterize the structure and acidity of modified HZSM-5. Moreover, the different structure and acidity of catalysts influenced on the products distribution of the butene oligomerization to high-quality liquid fuels reaction were also analyzed.

\section{Experimental}

\subsection{Catalysts preparation}

The preparation of HZSM-5 zeolite: $1.53 \mathrm{~g}$ sodium hydroxide $(\mathrm{NaOH})$ was dissolved in $150 \mathrm{~mL}$ deionized water. Then, a certain amount of sodium aluminate $\left(\mathrm{NaAlO}_{2}\right), 55.32 \mathrm{~g}$ tetrapropylammonium hydroxide (TPAOH) and $71.93 \mathrm{~g}$ tetraethyl orthosilicate (TEOS) were added into the $\mathrm{NaOH}$ solution under magnetic stirring. The obtained solution was hydrolyzed at room temperature for $45 \mathrm{~h}$, and transferred into teflon-lined stainless-steel autoclave to crystallize at $150^{\circ} \mathrm{C}$ for $72 \mathrm{~h}$. Later, the product could be obtained by centrifugation, washed by deionized water until neutral, dried at 110 overnight and calcinated at $550^{\circ} \mathrm{C}$ for $5 \mathrm{~h}$ to get the Na-ZSM-5. The HZSM-5 were obtained through ion-exchange with aqueous $\mathrm{NH}_{4} \mathrm{NO}_{3}$ solution $(1 \mathrm{~mol} / \mathrm{L})$ at $60^{\circ} \mathrm{C}$ for $2 \mathrm{~h}$ by 3 times, followed by washed with deionized water, dried at $110^{\circ} \mathrm{C}$ overnight and calcinated at $550^{\circ} \mathrm{C}$ for 5 h.

The preparation of hemicellulose modified HZSM-5: a certain quality of hemicellulose (obtained from a viscose fiber factory of Tangshan Sanyou Chemi-cal Industry Co.) was dissolved to the system at the beginning and the mass fraction contents of hemicellulose were controlled at $0.4 \%, 0.8 \%, 1.6 \%$ and $2.4 \%$ in the solution. Other steps were consistent with the method in preparation of HZSM-5, and the final products were denoted as HZSM-5-Hc-1, HZSM-5-Hc-2, HZSM-5-Hc-3, and HZSM-5-Hc-4.

\subsection{Catalysts characterization}

X-ray diffractograms (XRD) were obtained employing Bruker D8 Advance X-ray diffractometer with $\mathrm{Cu} \mathrm{Ka}$ radiation $(30 \mathrm{kV}, 30 \mathrm{~mA})$ in the range of $2 \theta$ of $5^{\circ}-50^{\circ}$ at a scanning rate of $2 \% \mathrm{~min}$. Scanning electron microscopy (SEM) was carried out in Hitachi S-4800 microscope operated at an $\begin{array}{lllll}\text { accelerating voltage of } 10.0 & \mathrm{kV} & \text { Nitrogen }\end{array}$ adsorption/desorption isotherms were measured at $-196^{\circ} \mathrm{C}$ on a Micromeritics ASAP 2020 adsorption apparatus. Before measurement, the samples were degassed at $320^{\circ} \mathrm{C}$ for $4 \mathrm{~h}$, and then performed the $\mathrm{N}_{2}$ adsorption/desorption experiment at $-196^{\circ} \mathrm{C}$. Temperature-programmed desorption of ammonia $\left(\mathrm{NH}_{3}\right.$-TPD) was performed in an Auto-Chem II 2920 chemisorption analyzer, and the thermal conductivity detector (TCD) was used to record desorption curve synchronously. Fourier transform infrared (FT-IR) spectra were measured on a Nicolet 6700 FT-IR spectrometer. Before testing, the sample cell was evacuated to $10^{-2} \mathrm{~Pa}$ at $450^{\circ} \mathrm{C}$. After that, pyridine vapor was introduced into the cell at room temperature for equilibration and the spectra were recorded after evacuation at $150^{\circ} \mathrm{C}$ for $1 \mathrm{~h}$ to get the FT-IR spectra for pyridine adsorption (Py-IR).

\subsection{Evaluation on butene oligomerization}

The oligomerizaion of butene was carried out in a continuous flowed fixed-bed at $4.0 \mathrm{MPa}$ and $270^{\circ} \mathrm{C}$. $1.5 \mathrm{~g}$ catalyst (40-60 mesh) was mixed with the same volume of silicon carbide (40-60 mesh) to avoid local overheating. Before reaction, the catalysts were pre-treated at $400^{\circ} \mathrm{C}$ for $4 \mathrm{~h}$ in a nitrogen flow (20 mL/min). When catalysts bed was cooled to the reaction temperature, butene (in the liquid phase) was fed to an evaporator with $180^{\circ} \mathrm{C}$ by a Series II digital pump, and then introduced to the reactor by flowing nitrogen to start the reaction.

To avoid the condensed and stranded of heavy oil phase in the system, the products of oligomerization reaction were collected in a hot trap with $200^{\circ} \mathrm{C}$ and followed by a cold trap with ice water, to collect heavy oil and light quality liquid products, respectively. The liquid products from both hot and cold trap were analyzed by a GC (Agilent 7820) equipped with DB-1 chromatographic column and FID detector. The gas compositions were performed on an Agilent 7890A fitted with Porapak Q packed column, 5A zeolite packed column and 
HP-PLOT $\mathrm{Al}_{2} \mathrm{O}_{3}$ "S" capillary column. The liquid products of the butene oligomerization reactions are referred to naphtha, diesel and heavy fractions. These fractions were segmented through SIMDIS according to the cut points: naphtha $\left(\mathrm{C}_{5}-173.9^{\circ} \mathrm{C}\right)$, diesel $\left(173.9-391.1^{\circ} \mathrm{C}\right)$ and heavy fraction $\left(391.1-1000^{\circ} \mathrm{C}\right)^{[22]}$. In order to close mass balance, the non-condensable vapors were quantified by a wet gas flowmeter. Only experiments with mass balances (MB) of $95 \%$ or higher were considered for discussion.

\section{Results and discussion}

\subsection{Catalysts characterization}

\subsubsection{Structure characterization of the catalyst}

Figure 1 shows the XRD patterns of all samples. All zeolites with or without hemicellulose have peaks at $2 \theta$ of $7.9^{\circ}$, $8.8^{\circ}, 23.1^{\circ}, 23.9^{\circ}$ and $24.4^{\circ}$, which exhibit the characteristic peaks of MFI structure. It is noting that there is slight difference for the relative intensities of $2 \theta$ at $7.9^{\circ}$ and $23.1^{\circ}$ with increasing hemicellulose, and HZSM-5-Hc-2 shows the least relative intensities at $7.9^{\circ}$ and $23.1^{\circ}$. This may indicate that the addition of hemicellulose can affect the relative crystallinity of each crystal face. Sano et $\mathrm{al}^{[26]}$ considered $\mathrm{OH}$ groups in cellulose as favourable sites for zeolite crystallization, and believed the hydroxyl group played an important role in the formation and crystallization of molecular sieve. Valtchev et $\mathrm{al}^{[27]}$ also reported the high activity of cellulose in the zeolite crystallization due to the high abundance of reactive hydroxyl groups. It was inferred that hemicellulose as the polymer of several kinds of monosaccharide had a large number of hydroxyl groups and these hydroxyl groups could act as an intervention or promoter in the nucleation and growth of ZSM-5. So, in the process of forming the molecular sieve, these hydroxyl groups will have selective effect on the growth of different crystal surface and the relative crystallinity of different crystal surface changed.

The morphology SEM images of all samples are given in Figure 2. It can be seen from Figure 2 that the sample without hemicellulose HZSM-5(a) indicates irregular and hexagon particles coexisting, while HZSM-5-Hc-1(b) shows more hexagon particles with increasing hemicellulose content,. When the mass fraction of hemicellulose is $0.8 \%$, HZSM-5-Hc-2(c) shows the most orderly morphology with a maximum of the hexagon crystals. This phenomenon is consistent with the XRD result, illustrating that the different contents of hemicellulose could have various influence on some crystal surfaces and morphology structure of ZSM-5.
The textural structure properties of all samples characterized by $\mathrm{N}_{2}$ adsorption-desorption are shown in Table 1. The BET surface area of HZSM-5 is $364 \mathrm{~m}^{2} / \mathrm{g}$. With increasing content of hemicellulose, the BET surface areas of HZSM-5-Hc-1and HZSM-5-Hc-2 are 359 and $398 \mathrm{~m}^{2} / \mathrm{g}$, respectively. When the mass fraction of hemicellulose is $1.6 \%$, HZSM-5-Hc-3 has the largest BET surface area of $582 \mathrm{~m}^{2} / \mathrm{g}$. However, with further increase in the content of hemicellulose the BET surface area of HZSM-5-Hc-4 reduces to $389 \mathrm{~m}^{2} / \mathrm{g}$. At the same time, with increasing content of hemicellulose the mesoporous volumes of HZSM-5-Hc-1, HZSM-5-Hc-2 and HZSM-5-Hc-3 are $0.20,0.32$, and $0.44 \mathrm{~cm}^{3} / \mathrm{g}$, respectively, which become larger ordinally. However, with further increase in the content of hemicellulose, the mesoporous volume of HZSM-5-Hc-4 was reduced to $0.31 \mathrm{~cm}^{3} / \mathrm{g}$. It can be seen that hemicellulose can affect the BET surface area and mesoporous volume of ZSM-5, which may resulted from the macromolecular carbon skeleton structure of hemicellulose. The hemicellulose acts as carbon template in the zeolite synthesis process, leading to larger BET surface area and more mesopore structure produced. When the mass fraction of hemicellulose is $1.6 \%$, it can enlarge the BET surface area and mesopore structure significantly.

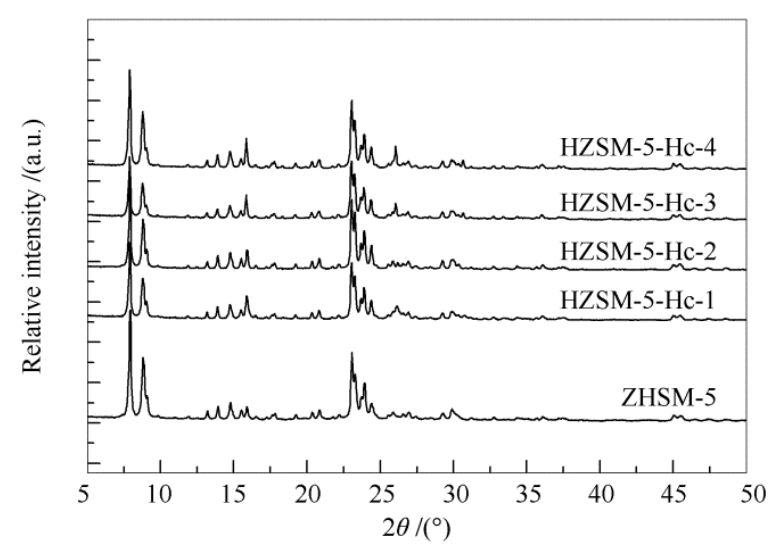

Fig. 1 XRD patterns of different zeolites

Table 1 Textural properties of different zeolites

\begin{tabular}{cccc}
\hline \multirow{2}{*}{ Catalyst } & \multirow{2}{*}{$S_{\mathrm{BET}}$} & \multicolumn{2}{c}{ Pore volume $v /\left(\mathrm{cm}^{3} \cdot \mathrm{g}^{-1}\right)$} \\
\cline { 3 - 4 } & $A /\left(\mathrm{m}^{2} \cdot \mathrm{g}^{-1}\right)$ & $v_{\text {micro }}$ & $v_{\text {meso }}$ \\
\hline HZSM-5 & 364 & 0.15 & 0.25 \\
HZSM-5-Hc-1 & 359 & 0.13 & 0.20 \\
HZSM-5-Hc-2 & 398 & 0.14 & 0.32 \\
HZSM-5-Hc-3 & 582 & 0.19 & 0.44 \\
HZSM-5-Hc-4 & 389 & 0.14 & 0.31 \\
\hline
\end{tabular}

note: $S_{\mathrm{BET}}$, BET surface area; $v_{\text {micro }}$, micropore volume determined by t-plot; $v_{\text {meso }}$, mesopore volume determined by $v_{\text {total }}-v_{\text {mico }}$ 
LI Chao et al / Journal of Fuel Chemistry and Technology, 2017, 45(9): 1088-1094
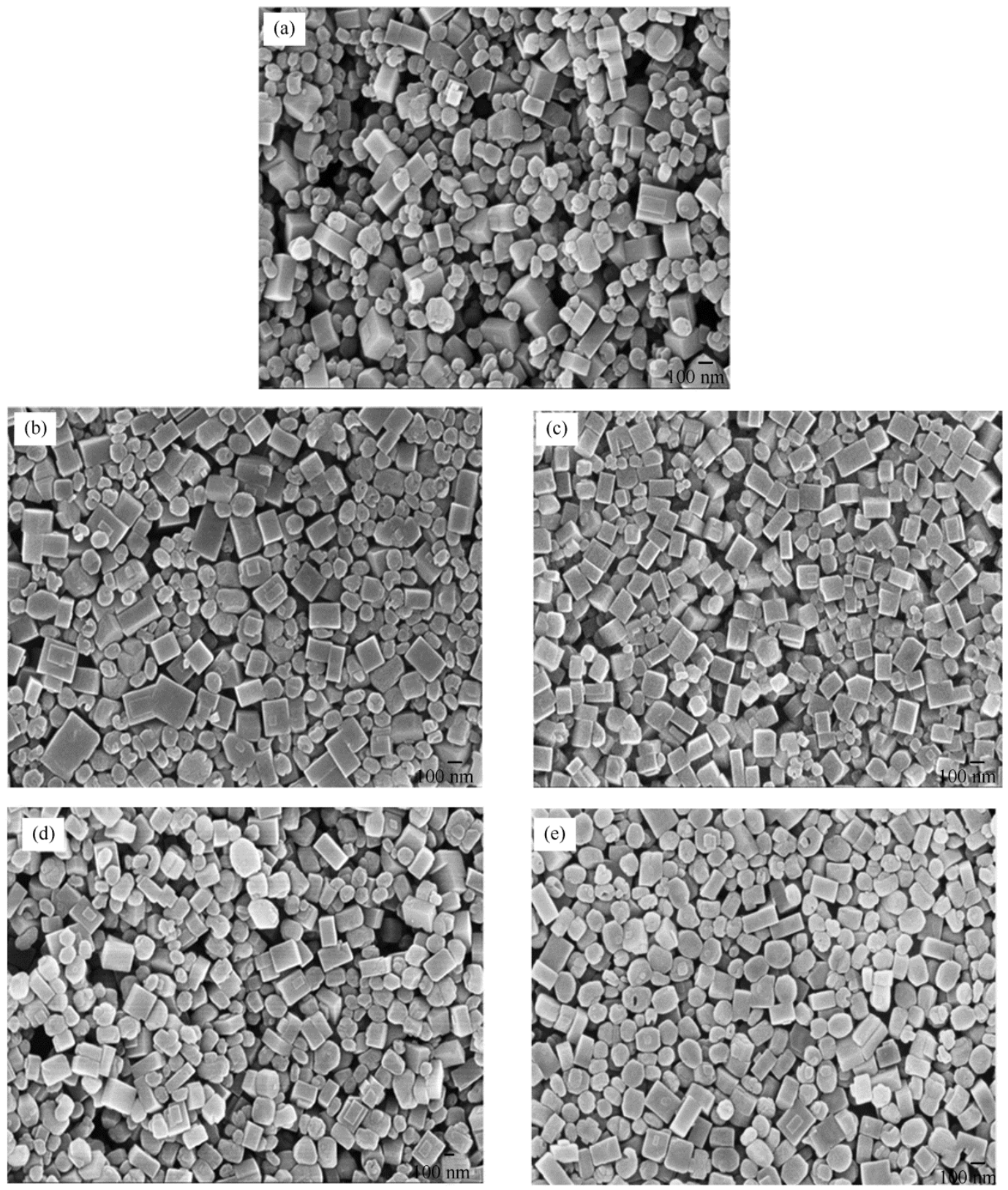

Fig. 2 SEM images of different zeolites

(a): HZSM-5; (b): HZSM-5-Hc-1; (c): HZSM-5-Hc-2; (d): HZSM-5-Hc-3; (e): HZSM-5-Hc-4

Table $2 \mathrm{NH}_{3}$-TPD and Py-IR results for different zeolites

\begin{tabular}{|c|c|c|c|c|c|}
\hline \multirow{2}{*}{ Catalysts } & \multicolumn{3}{|c|}{ Acidity by strength ${ }^{\mathrm{a}} /\left(\mathrm{mmol} \cdot \mathrm{g}^{-1}\right)$} & \multicolumn{2}{|c|}{ Acidity by type $\mathrm{e}^{\mathrm{b}} /\left(\mathrm{mmol} \cdot \mathrm{g}^{-1}\right)$} \\
\hline & weak & medium & total & Brønsted & Lewis \\
\hline HZSM-5 & 0.357 & 0.225 & 0.600 & 0.532 & 0.045 \\
\hline HZSM-5-Hc-1 & 0.512 & 0.467 & 0.979 & 0.809 & 0.049 \\
\hline HZSM-5-Hc-2 & 0.667 & 0.536 & 1.203 & 0.893 & 0.058 \\
\hline HZSM-5-Hc-3 & 0.859 & 0.621 & 1.480 & 1.209 & 0.075 \\
\hline HZSM-5-Hc-4 & 0.592 & 0.356 & 0.948 & 0.531 & 0.039 \\
\hline
\end{tabular}

a: density of the acid sites, assorted according to the acidic strength, determined by $\mathrm{NH}_{3}$-TPD. Medium, $\mathrm{NH}_{3}$ desorbed at $300-500^{\circ} \mathrm{C}$; weak, $\mathrm{NH}_{3}$ desorbed at $100-300^{\circ} \mathrm{C}$;

b: density of the acid sites, assorted according to the acidic type, determined by Py-IR 


\subsubsection{Acidity characterization of the catalyst}

In order to investigate the effect of hemicellulose on the acidity of HZSM-5, $\mathrm{NH}_{3}$-TPD and Py-IR are carried, as presented in Figure 3, and the quantitative results are listed in Table 2. There is only one distinct desorption peak of HZSM-5 at about $200^{\circ} \mathrm{C}$, indicating it mainly possess weak acidic sites and a small amount of medium acidic sites. With increasing content of hemicellulose the weak and medium strong acidic sites of HZSM-5-Hc-1, HZSM-5-Hc-2 and HZSM-5-Hc-3 gradually were enhance. However, as the content of hemicellulose increases further, the acid quantity of HZSM-5-Hc-4 was reduced. It is demonstrated that the addition of hemicellulose can improve the acidity of HZSM-5 in a certain level, and with increasing hemicellulose content, the acidity of catalyst has a tendency to increase first and decrease then. HZSM-5-Hc-3 has the strongest acidity.

Figure 4 shows the Py-IR spectra of all samples. The peaks at 1450 and $1520 \mathrm{~cm}^{-1}$ are corresponding to Lewis and $\mathrm{Br} \emptyset \mathrm{nsted}$ acid sites, respectively. The sharply peak at $1490 \mathrm{~cm}^{-1}$ is attributed to the vibration of the pyridine ring on Lewis and Brønsted acid sites. The result of Table 2 shows that the amount of the Brønsted and Lewis acid sites of HZMS-5 are 0.532 and $0.045 \mathrm{mmol} / \mathrm{g}$, respectively. With increasing hemicellulose content, the BrØnsted and Lewis acid sites of HZSM-5-Hc-1, HZSM-5-Hc-2 and HZSM-5-Hc-3 gradually enhance, while those of HZSM-5-Hc-4 decrease. Among all samples, HZSM-5-Hc-3 possesses the most quantity of BrØnsted and Lewis sites, being 1.209 and $0.075 \mathrm{mmol} / \mathrm{g}$, respectively, illustrating that the addition of hemicellulose can improve the acidity of HZSM-5 to some extent, especially for BrØnsted acid sites. With increasing content of hemicellulose, the acidity has a tendency to increase first and decrease then. HZSM-5-Hc-3 possesses the strongest acidity. This rule is fully consistent with the $\mathrm{NH}_{3}$-TPD results.

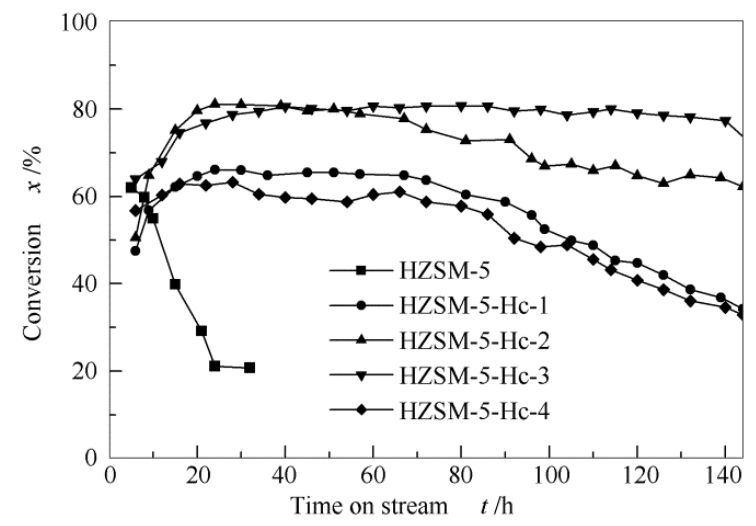

\subsection{Catalytic evaluation on butene oligomerization}

The catalytic results of butene oligomerization over all samples are shown in Figure 5 and the products collected after $24 \mathrm{~h}$ of time-on-stream at $270^{\circ} \mathrm{C}$ and $4 \mathrm{MPa}$ are analysed in Table 3.

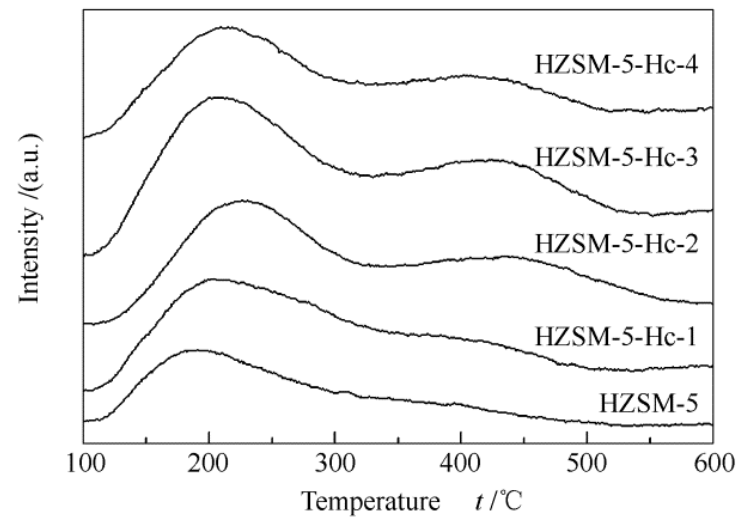

Fig. $3 \quad \mathrm{NH}_{3}$-TPD profiles of different zeolites

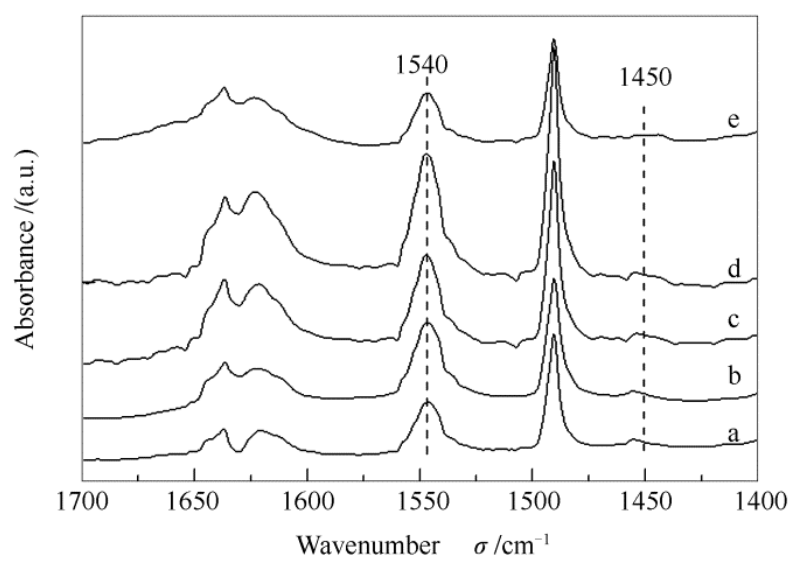

Fig. 4 Py-IR spectra of different zeolites

(a): HZSM-5; (b): HZSM-5-Hc-1; (c): HZSM-5-Hc-2; (d): HZSM-5-Hc-3; (e): HZSM-5-Hc-4

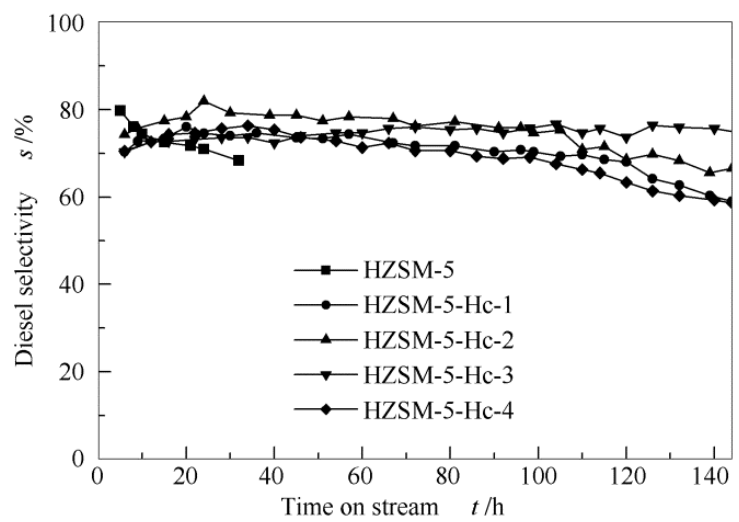

Fig. 5 Results of butene oligomerization with time on stream (TOS) over different catalyst 
Table 3 Catalytic results at $24 \mathrm{~h}$ for the butene oligomerization over different catalysts

\begin{tabular}{|c|c|c|c|c|c|c|c|}
\hline \multirow{2}{*}{ Catalyst } & \multirow{2}{*}{ Conversion $x / \%$} & \multicolumn{2}{|c|}{ Selectivity $s / \%$} & \multicolumn{4}{|c|}{ Carbon atom distribution $w / \%$} \\
\hline & & naphtha & diesel & $\mathrm{C}_{8}$ & $\mathrm{C}_{12}$ & $\mathrm{C}_{16}$ & $\mathrm{C}_{20+}$ \\
\hline HZSM-5 & 21 & 29 & 71 & 58 & 36 & 5 & 1 \\
\hline HZSM-5-Hc-1 & 66 & 25 & 75 & 49 & 41 & 7 & 2 \\
\hline HZSM-5-Hc-2 & 80 & 18 & 82 & 37 & 49 & 11 & 3 \\
\hline HZSM-5-Hc-3 & 79 & 26 & 74 & 29 & 38 & 19 & 14 \\
\hline HZSM-5-Hc-4 & 63 & 24 & 76 & 42 & 40 & 12 & 6 \\
\hline
\end{tabular}

The weight hourly space velocity (WHSV) is kept at $4.8 \mathrm{~g} /(\mathrm{g} \cdot \mathrm{h})$. As Figure 5 shown, the butene conversion on HZSM-5 without hemicellulose is $21 \%$ after $24 \mathrm{~h}$ of time-on-stream with the lowest conversion and poor activity. With increasing content of hemicellulose, the butene conversion on HZSM-5-Hc-1, HZSM-5-Hc-2, and HZSM-5-Hc-3 gradually improved. The conversion on HZSM-5-Hc-1 is $66 \%$ at $24 \mathrm{~h}$, which was higher than HZSM-5 obviously. HZSM-5-Hc-2 is further improved on the basis of HZSM-5-Hc-1. The conversion is up to $80 \%$ at $24 \mathrm{~h}$ although the overall activity is better than HZSM-5-Hc-1, while the stability needs to be further improved. The conversion on HZSM-5-Hc-3 is $79 \%$ at $24 \mathrm{~h}$, but it increases steadily to $80 \%$ until $36 \mathrm{~h}$ and remained after $140 \mathrm{~h}$, which also has obvious advantages on overall catalytic capacity compared with HZSM-5-Hc-2. However, as the content of hemicellulose increases further, the catalytic ability declines and the conversion on HZSM-5-Hc-4 decreases to $63 \%$ even lower than HZSM-5-Hc-2. This phenomenon is good enough to verify the acidity results of different contents of hemicellulose modified HZSM-5. HZSM-5-Hc-3 shows the best acidity and stability on butene oligomerization with the strongest acidity, which consistent with the literatures.

The liquid products of reaction over all samples are investigated by GC analysis. As for the HZSM-5 catalyst without hemicellulose, the product distribution is mainly composed of dimer (58\%) and trimer $(36 \%)$. When the hemicellulose is introduced, the selectivity of dimer reduces gradually and then increases with increasing concentration of hemicellulose. The liquid product of reaction over HZSM-5-Hc-3 has the most proportion of three and more polymerized products, as well as the most abundant of diesel components. This may related to its largest mesoporous volume and the strongest acidity.

\section{Conclusions}

In summary, the hemicellulose modified HZSM-5 catalyst was successfully prepared and the catalytic activity of prepared HZSM-5 was evaluated on butene oligomerization reaction. All the results showed that the content of hemicellulose had great effect on the structure, acidity, catalytic activity and stability of HZSM-5. When the mass fraction of hemicellulose was $0.8 \%$, HZSM-5-Hc-2 showed the most orderly morphology with a maximum of the hexagon crystals. When the hemicellulose was $1.6 \%$, HZSM-5-Hc-3 had high BET surface and mesoporous volume, the strongest acidity, and the best activity and stability on butene oligomerization. The butene conversion on HZSM-5-Hc-3 remained at about $80 \%$ with the most of trimer and tetramer products, and the selectivity of diesel was also the highest during $140 \mathrm{~h}$ time on stream. Thus, the addition of hemicellulose can affect the morphology, textural structure properties and acidity of HZSM-5 to some degree. Proper content of hemicellulose can improve the mesoporous volume, acidity as well as the catalytic ability of HZSM-5 on light of olefin oligomerizaton significantly.

\section{References}

[1] Zhang L M. Chain's foreign-trade dependence of crude oil more than 65\%. Econ Info Dai, 2017,01(006): 1.

[2] Kim Y T, Chada J P, Xu Z, Pagan-torres J, Rosenfeld D C, Winniford W L, Schmidt E, Huber G W. Low-temperature oligomerization of 1-butene with H-ferrierite. J Catal, 2015, 232: 33-44.

[3] Zhong L S, Yu F, An Y L, Zhao Y H, Sun Y H, Li Z J, Lin T J, Lin Y J, Qi X Z, Dai Y Y, Gu L, Hu J S, Jin S F, Shen Q, Wang H. Cobalt carbide nanoprisms for direct production of lower olefins from syngas. Nature, 2016, 538(7623): 84-87.

[4] Ji H, Lv Y J, Hu J X, Xiang H W, Li Y W. Advances in the catalytic reaction of olefin oligomerization. Prog Chem, 2002, 14(2): 146-155.

[5] Su X, Duan H M, Huang Y Q, Wang X D, Zhang T. Progress on the catalytic oligomerization of light olefins to liquid fuel. Prog Chem, 2016, 35(7): 2046-2056.

[6] Li X, Jiang X. Propylene oligomerization to produce diesel fuel on Zr-ZSM-5 catalyst. Chem Tech Fuels Oil, 2013, 49(2): 156-164.

[7] Coelho A, Caeiro G, Lemos M A N D A, Lemos F, Ribeiro F R. 1-Butene oligomerization over ZSM-5 zeolite: Part 1-Effect of reaction conditions. Fuel, 2013, 111: 449-460. 
[8] Ipatieff V N, Corson B B, Egloff G. Polymerization, a new source of gasoline. Ind Eng Chem, 1935, 27(9): 1077-1081.

[9] Wulfers M J, Lobo R F. Assessment of mass transfer limitations in oligomerization of buteneat high pressure on H-beta. Appl Catal A: Gen, 2015, 505: 394-401.

[10] Tabak S A, Krambeck F J, Garwood W E. Conversion of propylene and butylene over ZSM-5 catalyst. AIChE J, 1986, 32(9): 1526-1531.

[11] Quann R J, Green L A, Tabak S A, Krambeck F K. Chemistry of olefin oligomerization over ZSM-5 catalyst. Ind Eng Chem Res, 1988, 27(4): 565-570.

[12] Chen W Z. The development of composite technology and comparison. Pet Process Petroche, 1988(2): 16-21.

[13] Song R Q, Xiang H W, Li Y W, Zhong B. Alkene oligomerization to liquid fuels. J Fuel Chem Technol, 1999, 27: 79-89.

[14] Zhang X, Wang J W, Zhong J. Advance in catalysts and technology for butene oligomerization. Petrochem Technol, 2004, 33(3): 270-276.

[15] Li X M. Han D Z, Wang H, Liu G B, Wang B, Li Z, Wu J H. Propene oligomerization to high-quality liquid fuels over Ni/HZSM-5. Fuel, 2015, 144: 9-14.

[16] Coetzee J H, Mashapa T N, Prinsloo N M. Rademan J D. An improved solid phosphoric acid catalyst for alkene oligomerization in a Fischer-Tropsch refinery. Appl Catal A: Gen, 2006, 308: 204-209.

[17] Bellussi G, Mizia F, Calemma V, Pollesel P, Millini R. Oligomerization of olefins from Light Cracking Naphtha over zeolite-based catalyst for the production of high quality diesel fuel. Micropor Mesopor Mat, 2012, 164: 127-134.

[18] Behr A, Rentmeisiter N, Seidensticker T, Vosberg J, Stephan P, Maschmeyer D. Highly selective dimerization and trimerization of isobutene to linearly linked products by using nickel catalysts. Chemistry-ASIAN J, 2014, 9(2): 596-601.

[19] O'connor C T, Kojima M. Alkene oligomerization. Catal Today, 1990, 6(3): 329-349.

[20] Popov A G, Fedosov D A, Ivanova I I, Vedernikov O S, Kleimenov A V, Kondrashev D O, Miroshkina V D, Abrashenkov P A, Kuzentsov S E, A ZSM-5 zeolite-based catalyst for oligomerization of the butane-butylene fraction. Petrol Chem, 2016, 56(3): 237-243.

[21] Quann R J, Green L A, Tabak S A, Krambeck F J, Chemistry of olefin oligomerization over ZSM-5 catalyst. Ind Eng Chem Res, 1988, 27(4): 565-570.

[22] Corma A, Martinez C, Doskocil E, Designing MFI-based catalysts with improved catalyst life for oligomerization to high-quality liquid fuels. J Catal, 2013, 300: 183-196.

[23] Emdadi L, Wu Y, Zhu G, Chang C, Fan W, Pham T, Lobo R F, Liu D. Dual template synthesis of meso-and microporous MFI zeolite nanosheet assemblies with tailored activity in catalytic reactions. Chem Mater, 2014, 26(3): 1345-1355.

[24] Lin L, Qiu C, Zhuo Z, Zhang D, Zhao S, Wu H, Liu Y, He M. Acid strength controlled reaction pathways for the catalytic cracking of 1-butene to propene over ZSM-5. J Catal, 2014, 309: 136-145.

[25] Du J, Li B, Li C, Zhang Y D, Yu G, Wang H S, Mu X D. Tough and multi-responsive hydrogel based on the hemicellulose from the spent liquor of viscose process. Int J Bioll Macromol, 2016, 88: 451-456.

[26] Scott G, Thompson R W, Dixon A G, Sacco A. The role of triethanolamine in zeolite crystallization. Zeolites, 1990, 10(1): 44-50.

[27] Valtchev V, Mintova S, Vulchev I, Lazarova V. Influence of reactive radicals in cellulose fibres on the formation of zeolite coatings. J Chem Soc, Chem Commun, 1994, (18): 2087-2088. 